\title{
L'ÉVALUATION PSYCHOLOGIQUE EN CONTEXTE JUDICIAIRE : DÉNI OU SOUTIEN DE LA SUBJECTIVITÉ ?
}

Pascal Roman

Martin Média | «Le Journal des psychologues »

$2017 / 7 n^{\circ} 349$ | pages 46 à 50

ISSN 0752-501X

Article disponible en ligne à l'adresse :

https://www.cairn.info/revue-le-journal-des-psychologues-2017-7-page-46.htm

\section{Pour citer cet article :}

Pascal Roman, «L'évaluation psychologique en contexte judiciaire : déni ou soutien de la subjectivité ? », Le Journal des psychologues 2017/7 (n³49), p. 46-50. DOI 10.3917/jdp.349.0046

Distribution électronique Cairn.info pour Martin Média.

(c) Martin Média. Tous droits réservés pour tous pays.

La reproduction ou représentation de cet article, notamment par photocopie, n'est autorisée que dans les limites des conditions générales d'utilisation du site ou, le cas échéant, des conditions générales de la licence souscrite par votre établissement. Toute autre reproduction ou représentation, en tout ou partie, sous quelque forme et de quelque manière que ce soit, est interdite sauf accord préalable et écrit de l'éditeur, en dehors des cas prévus par la législation en vigueur en France. Il est précisé que son stockage dans une base de données est également interdit. 


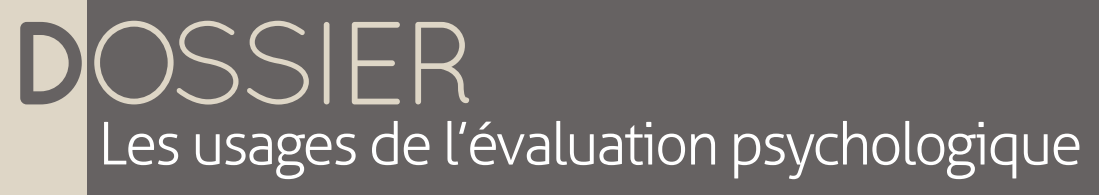

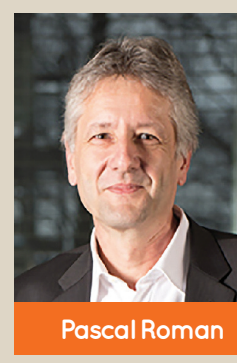

Professeur de psychologie clinique, psychopathologie et psychanalyse, laboratoire

LARPsyDIS, Institut de psychologie, université

de Lausanne (Suisse)

\section{L'évaluation psychologique déni ou soutien de la}

Parmi les pratiques d'évaluation dans lesquelles se trouvent engagés les psychologues, le champ de l'expertise judiciaire occupe une place à part. Face aux enjeux de pouvoir inhérents à cette pratique et aux risques d'instrumentalisation qui la sous-tendent, comment une expertise psychologique judiciaire peut-elle contribuer au soutien de la subjectivité du sujet de l'expertise ? Une prise de position clinique, technique ainsi qu'éthique et politique de la part du psychologue est un prérequis nécessaire.

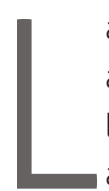

a question de l'évaluation se trouve aujourd'hui au cœur des processus démocratiques, avec les effets délétères que le recours systématisé et aveugle à l'évaluation contient, et les risques qu'il fait courir pour les libertés individuelles et la dynamique du contrat social (Abelhauser, Gori, Sauret, 2011).

En effet, la multiplication des démarches évaluatives tend, d'une part, à vider de son sens l'activité elle-même au profit de l'investissement de procédures dont la finalité au service de la « tâche primaire » (Kaës, 1987) se perd, et, d'autre part, à légitimer par un discours d'expert (ou de pseudo-expert) des décisions dans différents champs de la vie sociale (économique, gestionnaire, politique, judiciaire...).

C'est dans ce contexte que la question de la place de l'évaluation dans le champ judiciaire peut être posée, en particulier autour de la pratique de l'expertise psychologique judiciaire (expertise médico-psychologique lorsque l'expertise judiciaire associe médecin-psychiatre et psychologue). Cette question sera abordée à partir de trois points de vue:
- du point de vue clinique, en référence à la place de l'expertise judiciaire dans la pratique du psychologue, aux engagements et aux limites qu'elle mobilise ;

- du point de vue technique, en lien avec la mise en œuvre des compétences spécifiques du psychologue ;

- du point de vue politique, au regard des attentes sociales, voire des injonctions au cœur desquelles l'expert se trouve saisi.

L'enjeu, en arrière-plan, consistera à interroger la place et la fonction de la pratique évaluative de l'expertise judiciaire dans la rencontre avec le « sujet de l'expertise judiciaire » (Roman, 2007), au sein de la tension entre déni et soutien de la subjectivité. En d'autres termes, il s'agira de poser les questions suivantes: de quelle manière cette pratique contribue-t-elle au contrôle social en proposant, en miroir de l'auteur d'actes transgressifs, une forme de déni de la subjectivité de l'autre ? Et de quelle manière cette pratique peut-elle, de manière alternative, être conçue au bénéfice du sujet, dans une fonction de soutien de la subjectivité ?

\section{L'EXPERTISE JUDICIAIRE DANS LA PRATIOUU CLINIOUUE DU PSYCHOLOGUE}

Si la démarche de l'évaluation appartient de plein droit à la pratique du psychologue clinicien (et, de fait, à sa formation), avec en particulier la pratique du bilan psychologique, l'évaluation dans le cadre de l'expertise judiciaire constitue une pratique spécifique, qui engage un certain nombre d'enjeux auxquels le psychologue n'est pas nécessairement préparé dans le cadre de sa formation théorique, méthodologique et pratique. Un des points de butée principaux de la pratique de l'évaluation dans le champ de l'expertise judiciaire consiste dans l'écart radical qu'elle instaure au regard du projet de l'acte clinique d'évaluation:

- dans le champ du soin, l'évaluation psychologique (le bilan psychologique) réalisée par le psychologue à l'initiative 


\section{en contexte judiciaire : subjectivité?}

d'un médecin-psychiatre, d'une équipe pédagogique ou éducative... a pour objectif d'apporter les éléments en mesure de soutenir une orientation dans les prises en charge thérapeutique, éducative, pédagogique, sociale ; on peut ajouter que l'évaluation se trouve portée par le sujet concerné par l'évaluation (ou par son environnement) à travers l'énoncé d'une demande, même si le statut de celle-ci mérite toujours d'être interrogé (Roman, 2016b) ; - dans le champ judiciaire, l'évaluation psychologique (l'expertise judiciaire) est sollicitée par un tiers, représenté par la figure du magistrat et sur le mode de l'injonction, quelle que soit l'instance concernée (au civil pour les affaires familiales et la protection de l'enfant, au pénal pour les affaires délictueuses ou criminelles, du point de vue de l'auteur ou de la victime), dans l'objectif de soutenir la décision judiciaire ${ }^{1}$; dans ce contexte, la demande échappe en quelque sorte au sujet de l'expertise, puisqu'elle est portée par un tiers ${ }^{2}$ : le psychologue en charge de la réalisation de l'expertise se trouve alors invité à soutenir chez le sujet concerné la transformation d'une conception de l'expertise basée sur la seule contrainte en une démarche d'évaluation réalisée de manière contrainte, mais dont il pourra subjectivement tirer un bénéfice.

Du point de vue de l'identité et de la pratique professionnelle du psychologue, c'est le dispositif de l'examen psychologique qui constitue le point d'arrimage le plus consistant pour la pratique de l'expertise judiciaire. À la suite de Jean Guillaumin (1977), on peut considérer que cette pratique implique une approche dynamique et complexe du fonctionnement psychique du sujet rencontré, en appui sur un certain nombre d'outils spécifiques qui garantissent une possible objectivation des positions subjectives (Roman, 2015). L'examen psychologique, en effet, considéré dans la dynamique processuelle du sujet rencontré, autorise, au-delà des apports diagnostiques (du point de vue de l'efficience intellectuelle, du point de vue de l'inscription psychopathologique dans une nosographie...), la construction d'une compréhension du fonctionnement psychique.

\section{L'EXPERTISE JUDICIAIRE ET LA MISE À L'ÉPREUVE DES COMPÉTENCES DU PSYCHOLOGUE}

Comme je l'ai montré dans de précédents travaux (Roman, 1998 ; 2007), la situation de l'expertise judiciaire, psychologique ou médico-psychologique, se trouve saturée par un double enjeu, condensé dans la formulation du « pour-voir » du psychologue dans sa pratique de l'expertise judiciaire:

- le voir, avec un accent porté sur la mobilisation de la pulsion scopique (centration sur le voir, de l'acte, du sujet, et sur ses dires dans les procès-verbaux transmis...) au risque d'une économie de la référence à l'écoute du sujet et de sa souffrance ;

- le pouvoir que la désignation comme expert confère tout à la fois d'un point de vue factuel, dans la mesure de l'importance grandissante que semblent prendre les avis d'expert dans le procès judiciaire, et d'un point de vue imaginaire tant le vocable même d'expert, doublé du contexte de l'appartenance au judiciaire, vient exacerber $\rightarrow$

\section{Notes}

1. Il importe de noter que l'expert judiciaire, en France, quel que soit le cadre dans lequel il est requis et la spécialité qui est la sienne, est considéré dans sa mission comme un « auxiliaire de justice ».

2. La question de la demande dans le champ des pratiques judiciaires du psychologue mérite d'être interrogée et clarifiée, à la lumière, plus largement, des pratiques de consultation : cette question est complexe et mérite une attention spécifique dans toute rencontre clinique (Roman, 2016a) non seulement dans le cadre de sollicitation pour un bilan et-ou un accompagnement psychothérapeutique, mais également dans le cadre de l'expertise judiciaire. En effet, de même que l'on peut penser l'obligation judiciaire de soin comme une « offre contrainte de soin » (Roman, 2016b), de même l'expertise psychologique peut être considérée comme une « offre contrainte de rencontre et d'évaluation ». 


\section{DOSSIER \\ Les usages de l'évaluation psychologique}

$\rightarrow$ les mouvements de toute-puissance qui veillent au tréfonds de chaque individu, fût-il psychologue. Face au risque attaché à ce double enjeu, dont on entend bien qu'il se trouve sous-tendu par la mobilisation des pulsions partielles (dans le contexte d'une modalité prégénitale du fonctionnement psychique, en-deçà de l'accès à l'ambivalence), un certain nombre de points de repères techniques peuvent être profilés, dans l'objectif de garantir la qualité de l'engagement du psychologue dans la pratique

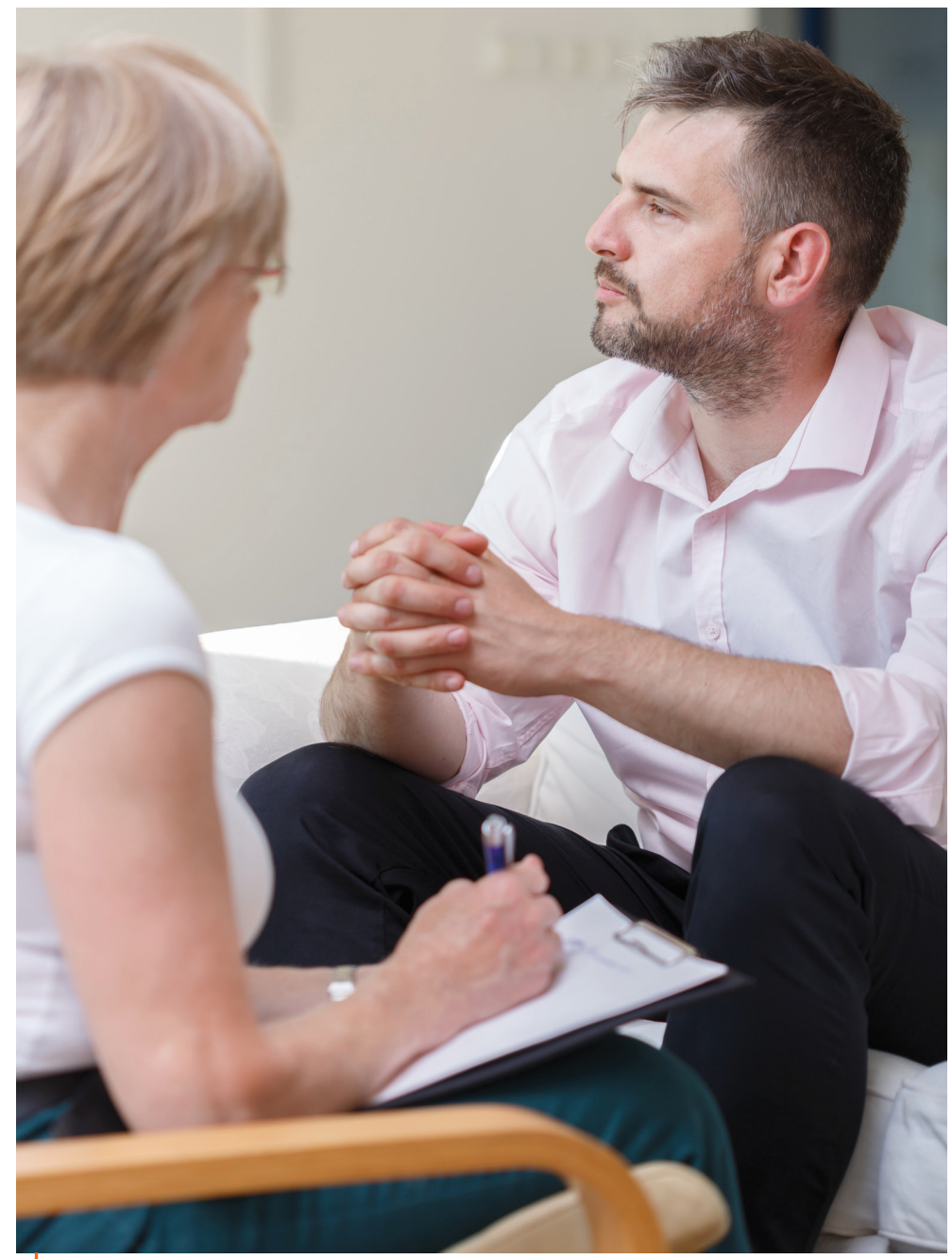

L'enjeu de l'évaluation est d'interroger la dynamique psychique dans laquelle l'acte commis ou subi s'inscrit. de l'expertise. En effet, le cœur des enjeux éthiques de la position du psychologue dans l'expertise judiciaire tient dans la mise en œuvre des conditions de dégagement d'un « voir séducteur », dans la lignée de ce que nomme Gérard Bonnet (1996) autour de la « violence du voir». Comme cela a été rappelé, tout, dans le processus auquel l'expert judiciaire prend part, engage au voir : la centration du libellé des ordonnances sur la référence au voir, mais aussi le voir lié à la communication de pièces de la procédure, y compris sous leur forme d'images (photos du délit ou du crime, enregistrement vidéoscopique de victimes mineures de violences sexuelles...), ainsi que la mise en scène du procès d'assises au cours duquel les scellés sont présentés face à la barre à laquelle se tient l'expert pour sa déposition... Le psychologue, dans sa mission d'expertise, prend ainsi une place de témoin, témoin d'un vu et-ou d'un entendu, dépositaire d'une part de vérité du sujet dont il aura à rendre compte. Comment sera-t-il en mesure de tenir cette tension, entre voir et dire, entre voir et ne pas tout dire? Comment le discours de l'expert peut-il se soutenir dans le regard du sujet de l'expertise pour échapper au risque disqualifiant et désubjectivant d'un discours qui parlerait d'un sujet sans adresse à celui-ci ? Afin de soutenir la nécessité de construire un dispositif de rencontre clinique autorisant un dégagement suffisant d'un voir séducteur, il est nécessaire d'introduire, de manière systématique dans la pratique de l'examen psychologique sous mandat judiciaire, la proposition d'épreuves projectives (particulièrement Rorschach et TAT, mais d'autres épreuves peuvent être retenues, particulièrement en clinique infantile) et-ou d'autres outils comme les questionnaires d'investigation clinique ${ }^{3}$ qui concourent à la compréhension du fonctionnement psychique du sujet (Roman, 2013a). Ces dispositifs cliniques, outils du psychologue par excellence, se présentent comme la proposition d'un support pour l'imaginaire, qui se déploie, paradoxalement, à partir d'une invitation au vu (épreuves projectives) ou à l'entendu (questionnaires). La confrontation à l'épreuve projective ou au questionnaire autorise ainsi l'expression des potentiels de symbolisation, tout comme celle des avatars du travail de symbolisation, dans un contexte où la parole spontanée, du fait de la dimension obligée de la rencontre et-ou du fait de la précarité des ressources symboliques, s'avère bien souvent empêchée.

Le décentrage de la focale de l'acte (ou du conflit) ainsi autorisé autour d'une expérience partagée en appui sur la médiation retenue permet d'ouvrir une autre scène, en contrepoint de la scène ouverte par l'instance judiciaire, qui se propose tout à la fois comme fondement à un travail d'appropriation subjective et comme ouverture d'un espace de jeu. C'est en appui sur cet espace de jeu, espace de symbolisation potentiel, que pourront tenter d'être formulés, de la part du clinicien, des éléments d'une compréhension des enjeux psychodynamiques de la participation du sujet dans une histoire de l'acte, permettant de réintroduire, de restaurer et-ou de soutenir la position du sujet de l'Inconscient, non réductible à l'acte. En d'autres termes, il s'agit d'offrir l'occasion au sujet de l'expertise d'une appropriation suffisante de la démarche d'investigation clinique initiée par la rencontre expertale, dans le contexte 
du caractère contraint de la rencontre. Si l'on peut considérer que cette proposition se présente sur un mode paradoxal (déployer un dispositif qui donne à voir ou à entendre, sur un mode potentiellement séducteur, pour se dégager du caractère séducteur de l'acte), sans doute ce paradoxe autorise-t-il l'instauration d'une ambiguïté suffisante au service de la rencontre clinique, à la condition qu'il se trouve contenu par l'investissement, de la part du psychologue, d'une posture clinique qui soutient l'émergence subjective. L'enjeu de l'évaluation dans le cadre de l'expertise judiciaire tient alors dans la nécessité d'interroger, au-delà de l'acte commis ou subi (ou du conflit dans lequel le sujet se trouve investi), la dynamique psychique dans laquelle celui-ci s'inscrit. Il s'agit de mettre l'accent sur la compréhension des modalités d'inscription de l'agir dans l'histoire des processus psychiques du sujet, dans la mesure où l'agir vient faire rupture dans ses aménagements et qu'il témoigne d'un mode de réaménagement dont il convient d'interroger le sens. Dans ce contexte, l'évaluation de la personnalité poursuivra un double projet:

- interrogation de la qualité de l'agir ou du conflit, agi ou subi, et du rapport entretenu avec celui-ci, particulièrement en termes de culpabilité ;

- interrogation de ce que l'on pourrait nommer « l'écho » de l'acte ou du conflit, en tant qu'il introduit une double mise en crise dans la vie intrapsychique (tant sur le plan narcissiqueidentitaire que sur le plan objectal-identificatoire) et dans les liens intersubjectifs.

\section{L'EXPERTISE JUDICIAIRE, LA POSTURE ÉTHIOQUE DU PSYCHOLOGUE ET SA CONTRIBUTION POLITIOUE}

On peut entendre, à partir de là, la nécessité pour le psychologue de clarifier ce qui fonde sa posture clinique dans sa pratique de l'expertise judiciaire. En effet, il importe de soutenir une affirmation éthique à même de contenir le risque de dérive perverse inhérente à la situation de l'expertise judiciaire. Cette affirmation éthique du psychologue s'inscrit dans une conception selon laquelle l'expertise psychologique judiciaire concerne la personnalité du sujet, considérée dans une compréhension subjective, inscrite dans un temps et des circonstances singulières (en tentant de se départir d'une vision totalisante et-ou définitive). La démarche de l'expertise psychologique judiciaire se propose alors dans une double exigence (Roman, 2007):

- elle se développe au service du soutien de la position du sujet, dans une attention à sa souffrance, mais aussi à ses potentiels de réaménagement et à ses ressources ;
- elle requiert, de la part de l'expert, une suspension du jugement, autorisant une écoute suffisamment dégagée des présupposés normatifs et moraux qui saturent l'inscription judiciaire du processus d'évaluation dans l'expertise. Cette position éthique, ancrée dans la posture professionnelle du psychologue, garantit le sujet de l'expertise judiciaire contre les risques d'instrumentalisation, judiciaire voire politique, dans laquelle le processus d'expertise pourrait le précipiter. En effet, cet enjeu se trouve tout particulièrement mobilisé dans la mesure où l'évaluation réalisée par le psychologue dans le contexte de l'expertise judiciaire concourt à la décision qui sera prise par le magistrat et-ou par l'instance de jugement. C'est sans doute là que la posture du psychologue en position d'expert judiciaire peut être décrite comme se situant sur le fil du rasoir. Comment, en effet, rendre compte du fonctionnement psychique du sujet, de ses inscriptions intersubjectives en lien avec l'instance judiciaire engagée, sans prendre un parti qui désignerait la voie à prendre en terme de décision? C'est sans doute la figure de l'ellipse qui est le plus à même de rendre compte de cette posture, en ce qu'elle interroge le dire du psychologue en position d'expert à destination de l'instance qui l'a mandaté. En arrière-plan, c'est la question du rapport à la confidentialité qui se trouve posée. Cette question de la confidentialité dans la pratique de l'expertise judiciaire apparaît comme incontournable (Roman, 2013b) et peut se décliner à partir de l'interrogation sur ce que l'expert pourrait avoir à transmettre, auprès de l'instance judiciaire, des propos, des pensées, des fantasmes... du sujet concerné. On le sait, la mission d'expertise est encadrée par un certain nombre de questions adressées au psychologue, auxquelles s'ajoute souvent une invitation adressée à l'expert d'apporter à la connaissance de l'instance judiciaire tous les éléments à même de « contribuer à la vérité ». Il s'agit alors de soutenir, à travers le rapport d'expertise et l'éventuelle déclinaison orale de celui-ci, une tension entre le dit et le non-dit (du sujet, du psychologue), en veillant à informer le sujet de l'expertise des contours du contenu de ce qui sera transmis au magistrat.

Au fond, on peut dire que l'enjeu de l'exercice de la confidentialité dans le cadre de l'expertise judiciaire tient moins à la codification formelle de ce qui pourrait ou non se dire, ou de quelle manière se dire (dire / ne

\section{Note}

3. On peut penser en particulier, dans le champ pénal, au questionnaire initié par Claude Balier et son équipe (Balier C., Ciavaldini A., Girard-Khayat M., 1996, Rapport de recherche sur les agresseurs sexuels, Direction générale de la santé), le QICPAAS, ou à son adaptation pour les adolescents, le OıcAAIcs (Roman, Ravit, 2010). 
$\rightarrow$ pas dire; dire / dire autrement), qu'à la promotion, à la construction et-ou à la restauration, par le lien clinique et au sein de celui-ci, d'une intimité fondatrice de la subjectivité. En d'autres termes, l'enjeu de l'exercice de la confidentialité dans le cadre de l'expertise judiciaire tient moins aux prescriptions déontologiques qu'à l'éthique du sujet et du soin, ou tient moins aux règles qu'à la posture clinique du clinicien qui prend le risque et accepte de s'engager dans une telle pratique... Ainsi, l'exercice de la confidentialité, et de la confiance qui la sous-tend, vise la transformation de la violence de l'exposition médiatique contenue dans la démarche d'expertise en une expérience de l'intime, dont seules des bribes, fragments d'une humanité partagée, pourront faire l'objet d'une transmission sur la scène judiciaire.

Sans doute la situation de l'expertise sollicitée pour soutenir une évaluation de l'évolution d'un sujet dans le cadre d'un mandat judiciaire de traitement condense-t-elle les enjeux de ce rapport à l'intime : en un temps où le pouvoir politique tente de limiter les espaces d'intimité entre patient et thérapeute dans le cadre des traitements sous contrainte, il s'avère urgent et nécessaire de défendre une suffisante disjonction des places et des fonctions. La démarche d'expertise menée de manière indépendante à l'égard de la démarche du soin permet de se départir de réponses en miroir de ce qui nourrit bien souvent la vie psychique des personnes concernées par l'expertise judiciaire en termes de confusion et de toute-puissance : en effet, une telle partition des interventions garantit le patient à l'égard des risques liés à la collusion des positions soignantes et expertales et garantit le clinicien à l'égard des fantasmes de toute-puissance auxquels ouvrirait droit une telle superposition.

En contrepoint, enfin, une précision mérite d'être apportée. La perspective qui valorise le soutien de la position

\section{Notes}

4. Cette question est, bien évidemment, beaucoup plus vaste ces dernières décennies ont conduit à revisiter l'idéologie de la demande qui, dans la fidélité à un vieil héritage freudien souvent non contextualisé, préside encore à certains discours cliniques sur le soin. Il est clair que bon nombre de situations cliniques concernent des sujets qui se trouvent empêchés dans l'accès à une demande de soin (problématiques narcissiques et limites, adolescence, addictions, violences...) ; c'est alors du point de vue de l'offre faite au sujet que le soin doit pouvoir être appréhendé, au risque de «non-assistance à personne (ou à société) en danger ».

5. Il serait intéressant d'interroger à cet égard le processus de désignation et de reconnaissance nominatif des experts en France, tandis qu'en Suisse cette pratique tend à s'exercer dans des structures spécialisées et en appui sur des formations complémentaires qui font l'objet de reconnaissance par les instances professionnelles. subjective, dans le contexte d'une rencontre contrainte comme celle de l'expertise psychologique judiciaire, ouvre à un renversement de paradigme dans le mode d'investissement de l'acte clinique par le psychologue. En effet, tout comme le psychothérapeute investi d'un mandat judiciaire de traitement est invité à penser le soin dans un en-deçà de la demande, en appui sur la valorisation d'une « offre contrainte de soin » (Roman, 2016b) ${ }^{4}$, le psychologue dans sa pratique de l'expertise judiciaire s'engage avec le sujet dans une offre de compréhension d'un pan de sa personnalité et de son histoire. Ainsi, le versant contraint de l'expertise se trouve-t-il transformé en un projet que l'on peut penser comme se déployant au plus près de l'intérêt subjectif du sujet.

\section{CONCLUSION}

Comme on a pu le montrer, la pratique de l'expertise judiciaire par le psychologue ne va pas de soi... et nécessite l'investissement d'une position d'écart suffisante à l'égard de l'instance judiciaire et des effets de séduction qu'elle peut être à même de mobiliser chez le professionnel. Cela vaut la peine de rappeler que tout comme le sujet inscrit dans un agir violent ne peut être réductible à son acte (il n'est pas d'« agresseur sexuel », mais « de sujet auteur d'agression sexuelle »), le psychologue engagé dans une pratique d'expertise judiciaire ne peut se résoudre à cette pratique à travers une désignation d'expert. En effet, l'expertise qui est reconnue au psychologue par les instances judiciaires ${ }^{5}$ ne prend de valeur que dans la mesure d'un certain nombre de conditions liées à sa pratique :

- elle est arrimée à une solide formation initiale (y compris dans le domaine juridique, tant civil que pénal) ;

- elle s'inscrit dans une confrontation régulière de sa pratique à celle de ses pair(e)s ;

- elle requiert une formation renouvelée tout au long de sa pratique ;

- elle se fonde sur une pratique clinique suffisamment large de l'évaluation et du soin (au-delà du champ psycholégal) qui lui permet de toujours mobiliser une posture d'écart, propre à soutenir une position éthique.

Dans le contexte si sensible des prises de position et des interventions expertales attendues du psychologue, ces différentes conditions constituent le minimum de ce que l'on peut attendre de celui qui s'engage dans une pratique d'expertise judiciaire, afin que celle-ci soit en mesure de se développer au service des processus de subjectivation, dont on sait qu'ils sont bien souvent mis à mal dans l'histoire du « sujet de l'expertise judiciaire ». 